\title{
MODELLING PEDESTRIAN LEVEL OF SERVICE ON SIDEWALKS WITH MULTI-FACTORS BASED ON DIFFERENT PEDESTRIAN FLOW RATES
}

\author{
Shinan SHU ${ }^{1}$, Yang $\mathrm{BIAN}^{2}$, Lin $\mathrm{ZHAO}^{3}$, Jian RONG ${ }^{4}$, Xiaoming $\mathrm{LIU}^{5}$ \\ 1,2,4,5Bijing Key Laboratory of Traffic Engineering, Beijing University of Technology, Beijing, China \\ ${ }^{3}$ National Center of ITS Engineering and Technology, Research Institute of Highway, \\ Ministry of Transport, Beijing, China
}

Submitted 19 June 2017; resubmitted 10 October 2017; accepted 25 April 2018

\begin{abstract}
Pedestrian Level of Service (PLOS) is influenced by the factors of traffic conditions, road facility conditions and environmental conditions. Pedestrian flow rate was the key factor influencing PLOS for the reason that pedestrians' visual scopes of pavement and the influencing degree of each influencing factor on sidewalks was differed under different pedestrian flow rates. In order to evaluate PLOS more accurately, this paper classified pedestrian flow rates into 6 stages. Then, significant influencing factors of traffic conditions, road facility conditions and environmental conditions, which influenced pedestrians' satisfaction, were extracted respectively under each pedestrian flow rate by Spearman rank correlation method. Finally, the evaluation method of PLOS with multi-factors based on classification of pedestrian flow rates was put forward. In addition, the models got training with fuzzy neural network method. The test showed that the accuracy of the comprehensive evaluation model of PLOS under different pedestrian flow rates based on fuzzy neural network reaches to $92 \%$, which is much higher than the model accuracy of previous researches.
\end{abstract}

Keywords: pedestrian level of service (PLOS), pedestrians' behaviour, pedestrians' satisfaction, pedestrian flow, evaluation model with multi-factors, fuzzy neural network.

\section{Introduction}

As a low-carbon travelling mode, walking has acquired more and more attention. A scientific evaluation method for Pedestrian Level of Service (PLOS) on sidewalks provides the most fundamental theoretical support for creating excellent walking environment. PLOS on sidewalks is a result of the joint influence of traffic conditions (Zhang et al. 2016; Kang et al. 2013; Dixon 1996), road facility conditions (Guo et al. 2014; Landis et al. 2005; Kadali, Vedagiri 2015) and environmental conditions (Khisty 1994; Muraleetharan, Hagiwara 2007). Thus, it is necessary to build an evaluation model with multi-factors for PLOS on urban sidewalks.

The initial PLOS on sidewalks was proposed by Fruin (1971). The PLOS was classified by establishing the relation model of pedestrian spacing, walking speed and pedestrian flow. The Traffic Engineering Manual (CHTS 1998) adjusted the classification criteria according to the actual road conditions in China. This kind of model only considered pedestrians' travelling demand from the perspective of traffic flow, while it neglected other influencing factors on pedestrians. By gathering all the influencing factors on pedestrians' satisfaction, Landis et al. (2001), Steiner et al. (2002), Petritsch et al. (2006), Sahani, Bhuyan (2017) and TRB (2010) established the relation models of pedestrians' satisfaction and the level of service of pedestrian facilities. However, these models were limited on modelling method because it neglected the difference among influencing factors of pedestrians' satisfaction under the conditions of different pedestrian density and degree of crowding. Meanwhile, the pedestrians' feeling obtained from questionnaires was very subjective so that the reliability of the model needed to be verified. Chen et al. (2017) considered road facility conditions and environmental conditions to evaluate pedestrian transportation facilities in Taiwan by using linear regression and support vector regression. Bian et al. (2007) comprehensively considered traffic conditions, road facility conditions and environmental conditions and established a linear regression model for evaluating PLOS

${ }^{*}$ Corresponding author. E-mail: bianyang@bjut.edu.cn

Copyright $\odot 2021$ The Author(s). Published by Vilnius Gediminas Technical University

This is an Open Access article distributed under the terms of the Creative Commons Attribution License (http://creativecommons.org/licenses/by/4.0/), which permits unrestricted use, distribution, and reproduction in any medium, provided the original author and source are credited. 
on Chinese sidewalks. However, linear regression model was only suitable for the regression analysis for continuous variables, while pedestrians' satisfaction ratings were ordinal variables. Petritsch et al. (2008) and Dowling et al. (2008) established models based on pedestrian density and other factors, respectively. The overall PLOS was the worse one of the 2 levels. This kind of model neglected the interacting effects among the influencing factors. The combined impact of traffic conditions, road facility conditions and environmental conditions could not be revealed.

Pedestrians had different psychological demand on the sidewalks under different road and traffic conditions (Wahba, Bridwell 1976; Sieben et al. 2017). In the case of large pedestrian volume or a narrow sidewalk, pedestrians were only interested in the most basic need which was the traffic need (Kim et al. 2014). In the case of small pedestrian volume or wide sidewalks, which meant that the basic need had been met, pedestrians would pay more attention on the comfort of road facility conditions and environmental conditions (Kang et al. 2013). Furthermore, pedestrians' range of vision also varied based on different pedestrian volumes (Roper, Hassan 2014). When there were a lot of pedestrians on the sidewalks, most of the road facility conditions and environmental conditions became invisible because of the occlusion by other pedestrians. Therefore, pedestrians might concern few factors. On the contrary, pedestrians' visual scope became wider with the decrease of pedestrian volume and pedestrians would concern more factors. It could be seen that pedestrian volume and the width of sidewalks were the basic influencing factors affecting PLOS on sidewalks. Pedestrian flow rate can present the result of the interaction of pedestrian volume and the width of sidewalks effectively, so that it could be considered as the basic influencing factor affecting PLOS on sidewalks. Under different pedestrian flow rate conditions, the factors that pedestrians concerned were different which meant that the influencing factors of PLOS on sidewalks were different. Therefore, this paper classified the levels of pedestrian flow rate according to the pedestrians' traffic states. On this basis, significant influencing factors of pedestrians' satisfaction were extracted. Then the evaluation index system and the evaluation model with the impact of multi-factors of PLOS on sidewalks could be established under each levels of pedestrian flow rate.

\section{Classification of pedestrian flow rates based on pedestrians' behaviours}

Classifying the levels of pedestrian flow rate was the basis of extracting significant influencing factors and establishing evaluation models for PLOS on sidewalks. This part classified pedestrian flow rates according to different pedestrian traffic states.

Some of the existing researches neglected pedestrians' psychological feeling. And some researches obtained pedestrians' feeling through pedestrians' satisfaction questionnaires, which was very subjective. It is generally known that Psychology determined behaviour and behaviour was the objective reflection of psychology (Kim et al. 2011). Then, pedestrians' psychological feeling could be obtained objectively through pedestrians' typical walking behaviours (Shan et al. 2016; Li et al. 2017). Thus, this paper captured pedestrians' typical walking behaviour under different pedestrian traffic states through video observation and image tracking method. In addition, the levels of pedestrian flow rate could be classified according to the corresponding relationship among pedestrians' traffic states, pedestrian walking behaviours and pedestrian flow rates.

Pedestrians' individual walking behaviour usually included normal walking, bypassing slower pedestrians, avoiding crossing conflicts and following others. In addition, these typical behaviours might appear independently or connectedly (Tang 2010). It could be discovered through observation that, under different pedestrian flow rates, when the above pedestrians' walking behaviours occurred, pedestrians' speeds and the relative lateral displacements differed. Thus, this research selected pedestrians' cumulative relative lateral displacement and pedestrians' speed as the classification criteria of pedestrian flow rates to determine the threshold of different levels of pedestrians' flow rate.

\subsection{Experiment designs and data collection}

For the reason that the sidewalks around subway stations and commercial area could cover all the conditions of pedestrian flow rates from free walking to congestion, video observation method was used to collect the image data of the sidewalk at B exit of Jingsong Subway Station and Xidan Commercial Area in Beijing (China). The Digital Video (DV) was set on a high spot outside the exit in order to cover the overall scene of the sidewalk along the parallel direction of the sidewalk. The observation area was $16 \mathrm{~m}$ long and $5 \mathrm{~m}$ wide in Jingsong Subway Station, and $14 \mathrm{~m}$ long and $5 \mathrm{~m}$ wide in Xidan Commercial Area. The survey time covered peak period and non-peak period in working days for covering the crowed situation. $1200 \mathrm{~min}$ of video segments were recorded in total, which covered various pedestrian flow conditions from free flow to congestion stream. Figure 1. showed the actual pedestrian flow scene of the observed sidewalk recorded by video. When the density of the pedestrian flow was high, the position of pedestrians' feet in the screen appeared superimposition. The actual position of pedestrians could be approximately estimated according to the position of their heads. In Figure 1, the yellow square at the bottom of the picture was the area in which pedestrians' feet fell (namely the observation region), and the red square above elaborated the corresponding area of the same pedestrians' heads.

Image tracking technology was used to capture pedestrians' walking trajectory, so as to collect pedestrians' speed and relative lateral displacements under different 

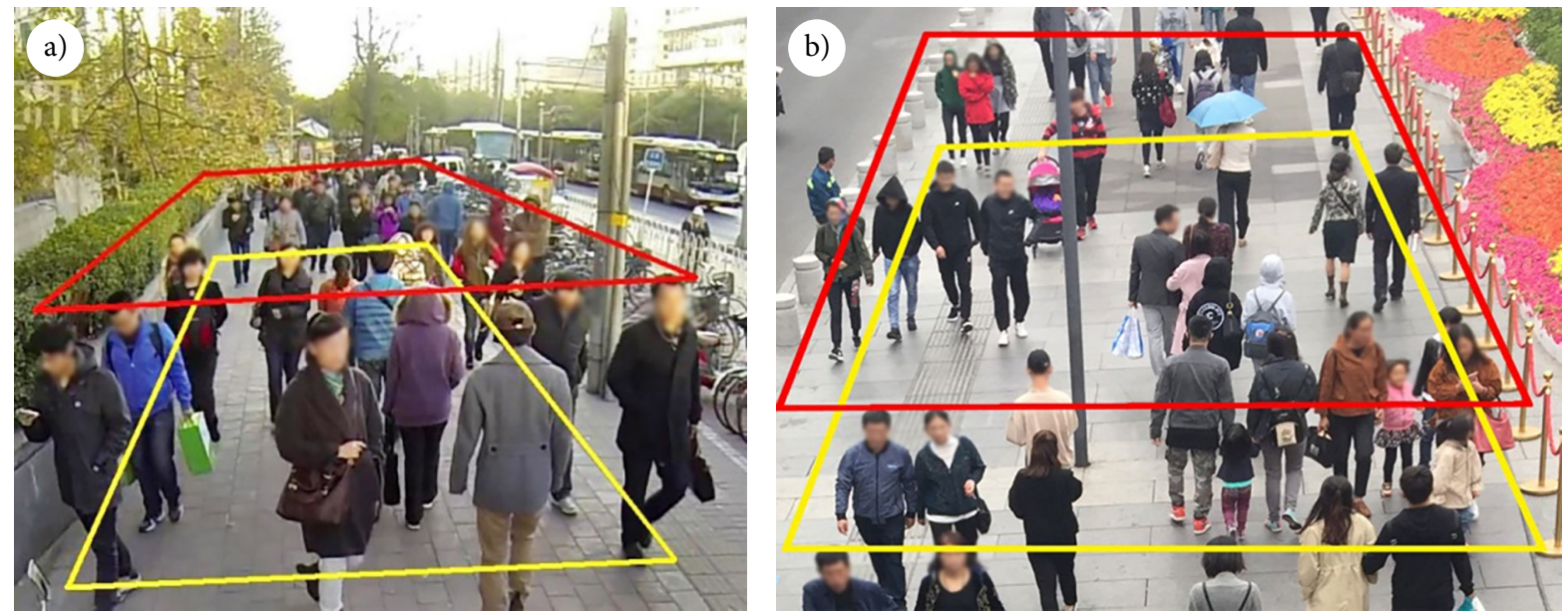

Figure 1. The actual pedestrian flow scene of the observed sidewalk: a - Jingsong Subway Station; b - Xidan Commercial Area

Table 1. Data of pedestrians' behaviour index

\begin{tabular}{|c|c|c|c|c|c|c|}
\hline \multirow{2}{*}{ Observing index of pedestrian behaviour } & \multicolumn{3}{|c|}{ Jingsong Subway Station } & \multicolumn{3}{|c|}{ Xidan Commercial Area } \\
\hline & minimum & maximum & mean & minimum & maximum & mean \\
\hline The cumulative relative lateral displacement in bypassing incident [m] & 0 & 3.59 & 0.99 & 0 & 3.56 & 0.98 \\
\hline The cumulative relative lateral displacement in avoiding incident $[\mathrm{m}]$ & 0 & 3.03 & 0.86 & 0 & 2.96 & 0.82 \\
\hline Pedestrians' speed $[\mathrm{m} / \mathrm{s}]$ & 0.34 & 1.64 & 1.12 & 0.40 & 1.62 & 1.09 \\
\hline
\end{tabular}

pedestrians' individual walking behaviours. Because of the limitation of the experimental site, the position of the DV could not be set right above the pedestrians. To this regard, the linear method of camera self-calibration from a single picture based on space coplanar lines (Fu et al. 2008) was taken to convert the plan coordinates of image to the actual scenes (namely the world coordinates). Then pedestrians' walking trajectory could be extracted. As a consequence, 780 groups of trajectory in the bypassing incidents and 1190 groups of trajectory in meeting incidents were obtained. The meeting incidents contained 2 conditions, i.e. avoiding crossing conflicts and following the queues. Table 1 showed the statistical result of pedestrians' behaviour index. It can be found that the pedestrians' behaviour index has no significant difference between Jingsong Subway Station and Xidan Commercial Area, although the traffic conditions and environmental conditions of these 2 places are different.

\subsection{Classifying the levels of pedestrian flow rate}

On the international, the classification criteria of 6 level proposed by TRB (2010) was usually used to evaluate PLOS. Therefore, this paper classified pedestrians' traffic states into 6 levels, and the thresholds of 6 levels of pedestrian flow rate could be confirmed.

Under the state of level A, pedestrian flow rate was low. Pedestrians' walking condition turned out to be a free flow. Pedestrians could walk along their desired direction and in their expected speed. When the bypassing incident or meeting incident happened, the relative lateral displacement between the observed pedestrians was 0 .
The typical pedestrians' behaviour trajectories under the state of level B to level D in Jingsong Subway Station and Xidan Commercial Area were shown in Figure 2. As the pedestrian flow rate change, the change rule of the pedestrians' behaviour trajectories was similar in these 2 places. In these circumstances, the difference between pedestrians' actual speed and their expected speed gradually increased. The interference and conflicts among pedestrians gradually increased. Pedestrian flow rate increased gradually until reaching the designed traffic capacity of the sidewalks. When the bypassing incident or meeting incident happened, the cumulative relative lateral displacements between the observed pedestrians gradually enlarged.

Under the state of level E to level F, it was almost impossible to bypass slower pedestrians. Bidirectional pedestrians walked on their certain routes with the shape of queuing. There was no need to avoid each other in meeting incidents and pedestrians' normal speeds were restricted. The pedestrians' flow rate increased continually on the basis of designed traffic capacity of sidewalks and their speeds decreased. After the speeds decreasing to the boundary of level $\mathrm{E}$ and level $\mathrm{F}$, then with the continually decrease of the speed, significant mutation and fluctuation appeared to the pedestrian flow rate. In addition, the typical queuing phenomenon appeared occasionally.

Based on the behaviour characteristics analysis above, the threshold of each level of pedestrian flow rate could be determined according to the variation ranges corresponding to the cumulative relative lateral displacements and speeds. Fuzzy clustering method was used to classify the levels for the 4 indexes of pedestrian flow rates, pedestrian speeds, cumulative relative lateral displacements in bypassing 
a)

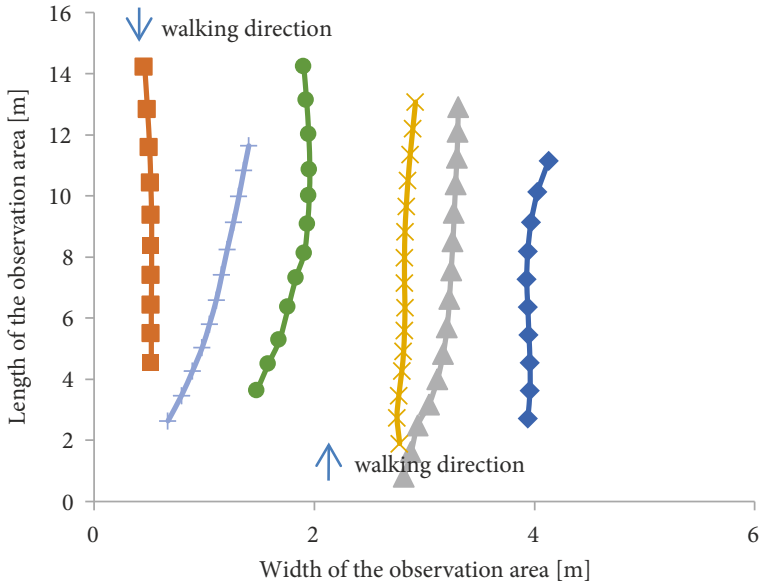

c)

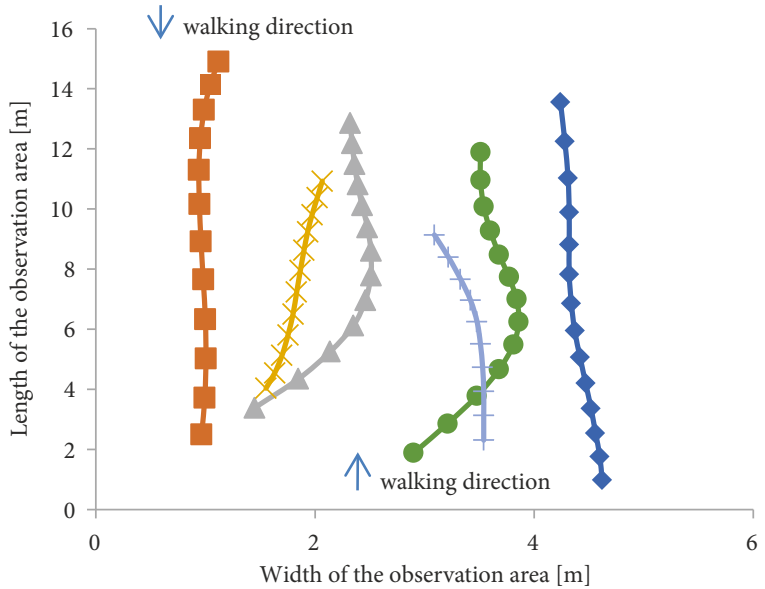

e)

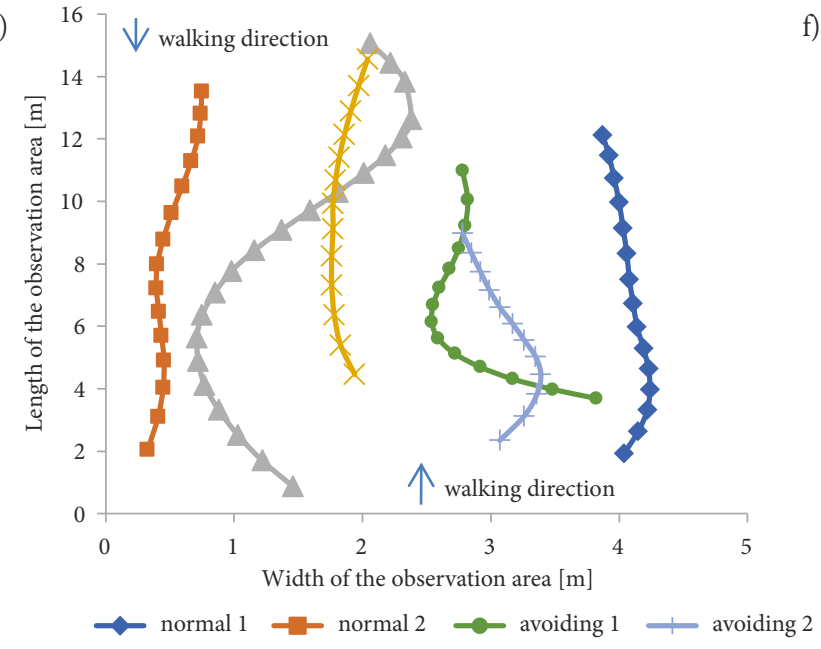

b)

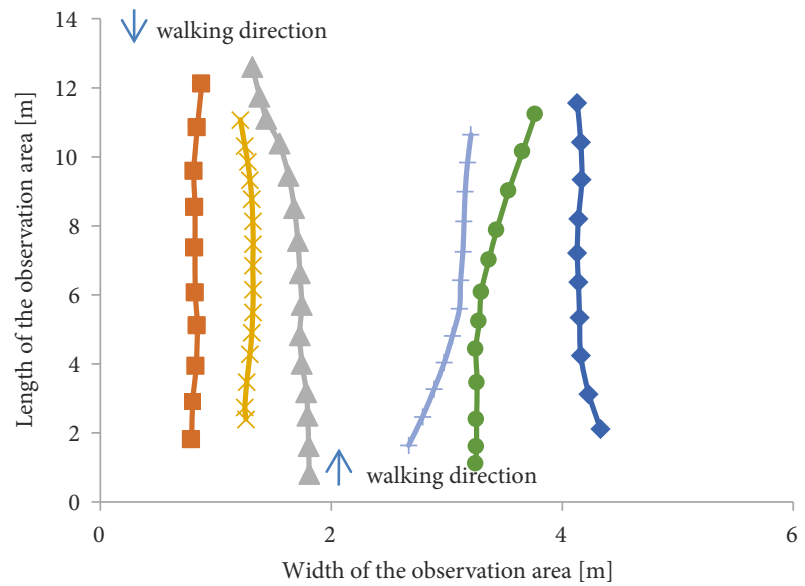

d)
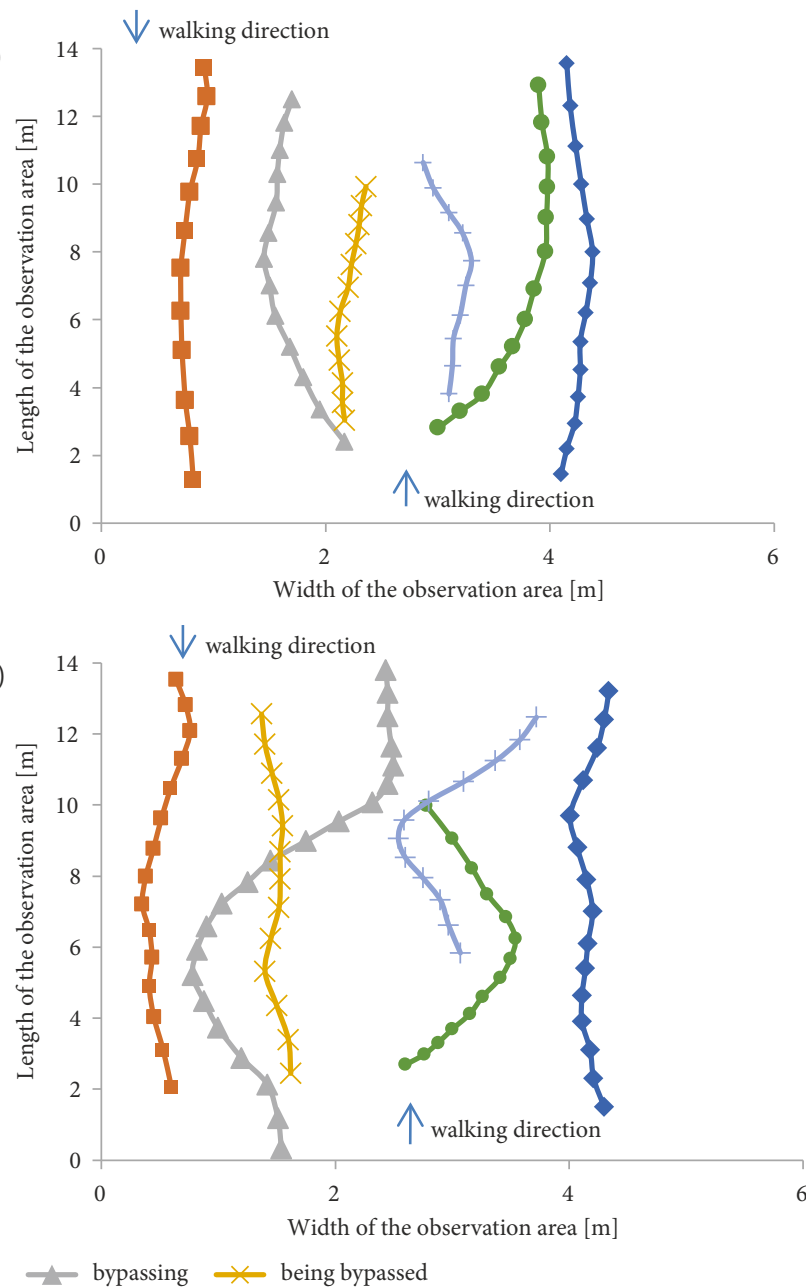

Figure 2. Typical behaviour trajectories under different levels of the pedestrian flow rate: a - at level B of the pedestrian flow rate in Jingsong Subway Station; b - at level B of the pedestrian flow rate in Xidan Commercial Area; c - at level C of the pedestrian flow rate in Jingsong Subway Station; $\mathrm{d}$ - at level C of the pedestrian flow rate in Xidan Commercial Area; e - at level D of the pedestrian flow rate in Jingsong Subway Station; $\mathrm{f}$ - at level D of the pedestrian flow rate in Xidan Commercial Area

incidents and avoiding incidents. The clustering centers obtained were the boundaries of pedestrian flow rate between each level. The calculating process was as follows.

Step 1. Building fuzzy matrix, which was the matrix data consisted with cumulative relative lateral displacement and speeds. It can be donated as:

$$
[A]=\left(X_{i j}\right), i=1,2,3 ; j=1,2, \ldots, m,
$$

where: the row of the matrix was each index value of $n$ groups of data.

Step 2. Data normalization, which was used to eliminate the magnitude difference between speeds and displacements. It can be calculated as:

$$
X_{i j}^{\prime}=\frac{x_{i j}-x_{j \min }}{x_{j \max }-x_{j \min }},
$$


where: $x_{i j}$ was the original matrix value; $X_{j \max }$ was the maximum of variable $j ; X_{j \text { min }}$ was the minimum of variable $j$.

Step 3. Building fuzzy relationship. The cosine method was used to build the similar matrix, which can be donated as:

$$
d_{i j}=\frac{\sum_{k=1}^{m} x_{i k} \cdot x_{j k}}{\sqrt{\sum_{k=1}^{m} x_{i k}^{2}}} \cdot \sqrt{\sum_{k=1}^{m} x_{j k}^{2}} .
$$

Step 4. Building the fuzzy equivalence matrix $\left[X^{n}\right]$, and the convolution computation was calculated on the fuzzy matrix: $[X] \rightarrow\left[X^{2}\right] \rightarrow\left[X^{3}\right] \rightarrow \ldots \rightarrow\left[X^{n}\right]$. After the limited times of convolution, make $\left[X^{n}\right] \cdot[X]=\left[X^{n}\right]$.

Step 5. Fuzzy C-means clustering method was used to determine the degree of each data belonging to the level through the membership. The clustering center of the 3 levels of pedestrian flow rate, which were level B to level D could be calculated through multiple iterations.

Since there was no relative lateral displacement at level A, level $\mathrm{E}$ and level F, the lower limit of level B of pedestrian flow rate was the observed value of the pedestrian flow rate when pedestrian cumulative relative lateral displacement infinitely tend to 0 . The upper limit of level D of pedestrian flow rate was the designed traffic capacity of sidewalks, which valued $40 \mathrm{ped} /(\mathrm{m} \cdot \mathrm{min})$.The upper limit of level $\mathrm{E}$ of pedestrian flow rate was the turning point from the increasing flow rate to appearing the fluctuation. Table 2 showed the threshold of each level of pedestrian flow rate and the range of behaviour indexes.

\section{Extracting significant influencing factors of pedestrians' satisfaction under different pedestrian flow rates}

The factors, which affected pedestrians' satisfaction varied under different levels of pedestrian flow rate. On the basis of trying to consider the comprehensive influencing factors of PLOS on sidewalks, the significant influencing factors under different pedestrian flow rates were extracted with Spearman rank correlation method.

\subsection{Experiment designs and data collection}

In order to make the conclusions more universally applicable, a large-scale survey was conducted on the sidewalks of different kinds of regions in Beijing. The cover of the questionnaire gave a detailed explanation of the objective of the survey. If pedestrians did not agree to take part in this survey, they could freely choose not to respond any questions without any consequences. Thus, we considered the receipt of a questionnaire to indicate informed consent. We did not obtain any data of personal identifiers and analysed the data anonymously. The study was reviewed and approved by the Ethics Committee of College of Life Science and Bioengineering, Beijing University of Technology (China).

Pedestrians' satisfaction survey was conducted with intercept survey method from the perspective of pedestrians' sense of safety and comfort. The form of the questionnaire scale was $1 \ldots 10$ ratings. Meanwhile, traffic conditions, road facility conditions and environmental conditions were also captured in the actual sidewalks. The data range of the quantitative variables was presented in Table 3 .

Table 2. The comparison of the threshold of each level of pedestrian flow rate and pedestrians' behaviour indexes

\begin{tabular}{|l|c|c|c|c|c|c|}
\hline PLOS & level A & level B & level C & level D & level E & level F \\
\hline Pedestrian flow rate [ped/(m·min)] & $<13$ & $13 \ldots 22$ & $22 \ldots 29$ & $29 \ldots 40$ & $40 \ldots 52$ & uncertainty \\
\hline $\begin{array}{l}\text { The cumulative relative lateral } \\
\text { displacement in bypassing incident [m] }\end{array}$ & 0 & $0 \ldots 0.77$ & $0.77 \ldots 1.66$ & $>1.66$ & 0 & 0 \\
\hline $\begin{array}{l}\text { The cumulative relative lateral } \\
\text { displacement in avoiding incident [m] }\end{array}$ & 0 & $0 \ldots 0.81$ & $0.81 \ldots 1.56$ & $>1.56$ & 0 & 0 \\
\hline Pedestrians' speed [m/s] & $>1.25$ & $>1.25$ & $1.25 \ldots 0.99$ & $0.99 \ldots 0.77$ & $0.77 \ldots 0.5$ & $<0.5$ \\
\hline
\end{tabular}

Table 3. Quantitative variables of traffic conditions, road facility conditions and environmental conditions

\begin{tabular}{|l|l|c|c|c|}
\hline \multicolumn{1}{|c|}{ Category of factors } & \multicolumn{1}{|c|}{ Name of factors } & Minimum & Maximum & Mean \\
\hline \multirow{5}{*}{ Traffic conditions } & pedestrian flow rate [ped/(m·min)] & 1 & 65 & 6 \\
\cline { 2 - 5 } & bicycle volume [veh/min] & 0 & 28 & 3 \\
\cline { 2 - 5 } & electric bike volume [veh/min] & 0 & 33 & 4 \\
\cline { 2 - 5 } & vehicle volume on the inside lane [veh/min] & 0 & 40 & 6 \\
\hline \multirow{2}{*}{ Road facility conditions } & valid width of sidewalks [m] & 0 & 4 & 1 \\
\cline { 2 - 5 } & frequency of barriers on the sidewalks [-] & 1 & 10 & 6.5 \\
\hline Environmental conditions & rating for the environment at the inner side of sidewalks [point] & \multicolumn{2}{|c|}{6} & \multicolumn{2}{|c|}{6} \\
\hline
\end{tabular}


Many of the influencing factors of pedestrians' satisfaction could not be quantitatively described so that the descriptive language was used to qualitatively describe them. As is shown in Table 4, the qualitative factors were described according to their categories, which were obtained through the field survey. The coding process of the qualitative factors was detailed in the next section.

Typical scenes of the survey were shown in Figure 3. In scene 1, the sidewalk located on the branch road of the residential area with walls of the community and greening inside. There were green buffer outside the sidewalk and on-street parking existed. Non-motorized vehicles and motorized vehicles mixed travelled. In scene 2 , the sidewalk located on the minor arterial of the commercial area with parking in front of the shopping mall and greening inside. There was no segregation outside the sidewalk. Non-motorized vehicles and motorized vehicles were segregated with scribing. In scene3, the sidewalk located on the arterial of the transportation hub area with the tall wall inside and fence outside. Non-motorized vehicles and motorized vehicles were segregated with fence. In scene 4 , the sidewalk located on the arterial of the general area with shops inside. There were greening buffers and parking (including parking on the sidewalk and on-street parking) outside. Non-motorized vehicles and motorized vehicles were segregated with grassland.

Table 4. Quantitative variables of road facility conditions and environmental conditions.

\begin{tabular}{|c|c|c|}
\hline Category of factors & Name of factors & Type of factors \\
\hline \multirow{3}{*}{$\begin{array}{l}\text { Road facility } \\
\text { conditions }\end{array}$} & road grade & expressway, arterial, minor arterial, branch road \\
\hline & $\begin{array}{l}\text { segregated facilities between pedestrians } \\
\text { and vehicles }\end{array}$ & $\begin{array}{l}\text { no segregation, on-street parking, fence, parking on sidewalks, } \\
\text { green buffer }\end{array}$ \\
\hline & $\begin{array}{l}\text { segregated facilities between non-motorized } \\
\text { vehicles and motor vehicles }\end{array}$ & $\begin{array}{l}\text { mixed traffic flow, segregated with scribing, segregated with } \\
\text { fence, segregated with grassland }\end{array}$ \\
\hline \multirow{2}{*}{$\begin{array}{l}\text { Environmental } \\
\text { conditions }\end{array}$} & $\begin{array}{l}\text { environment at the inner side of the } \\
\text { sidewalks }\end{array}$ & $\begin{array}{l}\text { the walls outside the buildings, shops, greening, walls, } \\
\text { construction plate, fences of the community, parking }\end{array}$ \\
\hline & regional classification & $\begin{array}{l}\text { residential area, commercial area, transportation hub area, } \\
\text { general area }\end{array}$ \\
\hline
\end{tabular}
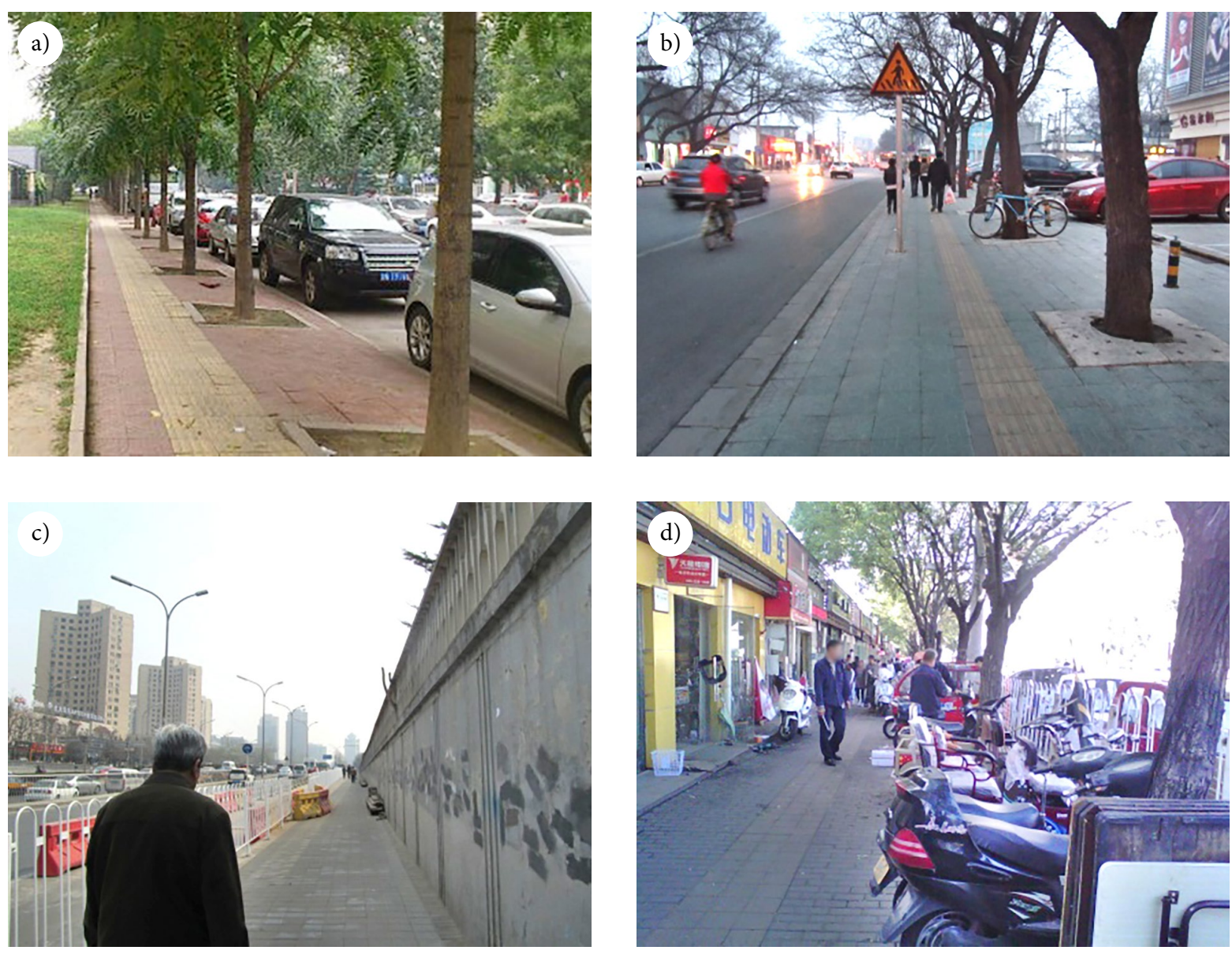

Figure 3. Actual scenes of survey sites: a - typical scene 1; b - typical scene 2; c - typical scene 3; $d$ - typical scene 4 
A pre-survey was conducted before the formal survey to calculate the acceptable sample size of formal survey. 302 valid questionnaires were obtained through pre-survey. The acceptable sample size can be calculated as follows:

$$
n=\left(\frac{t \cdot \sigma_{i}}{E}\right)^{2},
$$

where: $n$ is the acceptable sample size; $t$ is the corresponding value for $\alpha$-level; $\sigma_{i}$ is the standard difference for different levels of pedestrian flow rate, and $E$ is the sampling error.

As a result, $t=1.96(\alpha=0.05), E=0.2, \sigma_{A}=2.16$, $\sigma_{B}=1.64, \sigma_{C}=1.69, \sigma_{D}=1.28$, and $\sigma_{E}=0.68$. Therefore, the minimum sample size is calculated as: 448 - for level A, 258 - for level B, 274 - for level C, 157 - for level $\mathrm{D}$ and 44 - for level $\mathrm{E}$.

132 typical urban sidewalks were surveyed and 8000 valid questionnaires were obtained through formal survey. The number of questionnaires of different levels all exceeds the minimum sample sizes. The proportion of male respondents was $54 \%$ while that of female was $46 \%$. The ages of respondents contained all stages from below 20 to above 60 . Nearly $3 / 5$ of them were familiar with the surveyed sidewalks. The survey time covered 7:00 am to 7:00 pm on both working days and weekends.

\subsection{Extraction of significant influencing factors based on spearman rank correlation analysis}

Factors affecting pedestrians' satisfaction included traffic conditions, road facility conditions and environmental conditions, which were detailed in Tables 3 and 4. The segregations among pedestrians, non-motorized vehicles and motorized vehicles were both categorical variables, which could be converted to ordinal variables. The order of the segregation between pedestrians and vehicles could be arranged based on pedestrians' satisfaction with the Delphi method. In addition, the order of the segregation between non-motorized vehicles and motorized vehicles could be arranged based on the distances between pedestrians and motorized vehicles from the longer distances to the shorter ones. The influence of the environmental conditions was complicated and it impacted synthetically on pedestrians' satisfaction. So, $1 . .10$ satisfaction ratings were used to obtain pedestrians' satisfaction about the environment.

Since pedestrians' satisfaction grades and many influencing factors were all ordinal variables, the relationship between pedestrians' satisfaction and each influencing factor was analysed with the Spearman's rank correlation analysis method. Then the significant influencing factors could be extracted. Spearman's rank correlation coefficient was a non-parametric index for measuring the dependence between 2 variables. The correlation of the 2 statistics was evaluated with monotonous equation (Myers, Well 2003). If there was no duplicate values and the correlation of the 2 variables were complete monotonous, the Spear- man correlation coefficient was +1 or -1 . The correlation coefficient can be calculated as follows:

$$
\rho=\frac{\sum_{i}\left(x_{i}-\bar{x}\right) \cdot\left(y_{i}-\bar{y}\right)}{\sqrt{\sum_{i}\left(x_{i}-\bar{x}\right)^{2} \cdot \sum_{i}\left(y_{i}-\bar{y}\right)^{2}}} .
$$

The Spearman's rank correlation coefficients and the significant results between pedestrians' satisfaction ratings and each influencing factor under different grade of the pedestrian flow rate was calculated as shown in Table 5.

The following conclusions could be obtained from Table 5:

"» when the pedestrian flow rate was low (at level A and level B), all the factors of traffic conditions, road facility conditions and environmental conditions of sidewalks affected pedestrians' satisfaction significantly. The significance values between pedestrians' satisfaction and each factor were all below 0.05 ;

"» when the pedestrian flow rate increased till the high level (at level C and level D), the significant influencing factors decreased and the influence of road facility and environmental conditions weakened;

"» when the pedestrians flow rate was congestion (at level E), pedestrians almost paid no attention to the influencing factors except for pedestrian flow rate. The segregated facility between pedestrians and vehicles was the factor, which could prevent pedestrians from interference with other traffic flow and assure their safety. Thus, the influence of this factor was significant.

\section{Evaluation model of PLOS on sidewalks under different pedestrian flow rates}

In order to better describe the influencing degree of different factors and to present the integrated affecting result, a comprehensive evaluation model with multi-factors for PLOS on sidewalks was established based on different pedestrian flow rates, and the fuzzy neural network method was used to train it.

\subsection{Model of the fuzzy neural network}

The evaluation criterion of PLOS on sidewalks based on pedestrians' walking demand was pedestrians' satisfaction ratings. Pedestrians' satisfaction had the characteristic of randomness and fuzziness so that the traditional mathematics methods were difficult to stimulate the relationships between pedestrians' satisfaction and influencing factors. Hence, the fuzzy mathematics method was applied in evaluating PLOS (Liang 2006). However, fuzzy mathematics needed to select membership functions and fuzzy inferences artificially and it was absence of self- adaption ability. Thus, fuzzy neural network system was used to evaluate PLOS through training the input and output samples with the hybrid learning algorithm and identifying the parameters of membership functions and the accurate fuzzy coefficients. 
Table 5. Correlation between pedestrian satisfaction scores and each influencing factors under different pedestrian flow rates

\begin{tabular}{|c|c|c|c|c|c|c|c|c|c|c|c|c|}
\hline 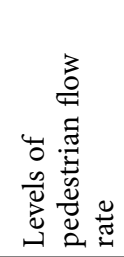 & 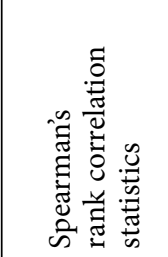 & 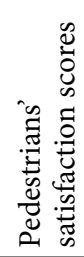 & 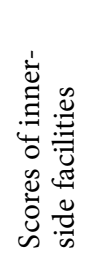 & 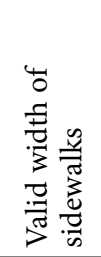 & 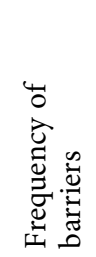 & 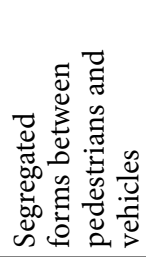 & 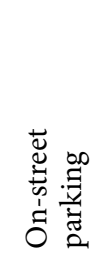 & 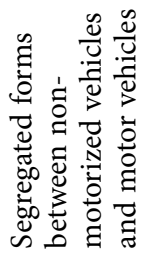 & 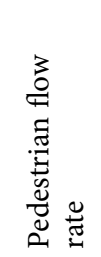 & 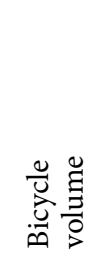 & 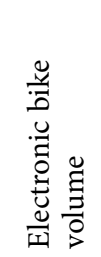 & 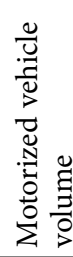 \\
\hline \multirow{3}{*}{ Level A } & $\begin{array}{l}\text { correlation } \\
\text { coefficient }\end{array}$ & 1.000 & 0.571 & 0.075 & -0.159 & 0.152 & -0.130 & 0.221 & -0.018 & -0.133 & -0.168 & 0.063 \\
\hline & \begin{tabular}{|l|} 
significance \\
(2-tailed)
\end{tabular} & - & 0.000 & 0.000 & 0.000 & 0.000 & 0.000 & 0.000 & 0.000 & 0.000 & 0.000 & 0.001 \\
\hline & sample size & 2684 & 2684 & 2684 & 2684 & 2684 & 2684 & 2684 & 2684 & 2684 & 2684 & 2684 \\
\hline \multirow{3}{*}{ Level B } & $\begin{array}{l}\text { correlation } \\
\text { coefficient }\end{array}$ & 1.000 & 0.683 & 0.058 & -0.106 & 0.398 & -0.274 & 0.269 & -0.092 & -0.023 & -0.280 & 0.078 \\
\hline & \begin{tabular}{|l|} 
significance \\
(2-tailed)
\end{tabular} & - & 0.000 & 0.000 & 0.000 & 0.000 & 0.000 & 0.000 & 0.001 & 0.000 & 0.000 & 0.138 \\
\hline & sample size & 722 & 722 & 722 & 722 & 722 & 722 & 722 & 722 & 722 & 722 & 722 \\
\hline \multirow{3}{*}{ Level C } & $\begin{array}{l}\text { correlation } \\
\text { coefficient }\end{array}$ & 1.000 & 0.515 & -0.408 & 0.268 & 0.320 & 0.236 & -0.390 & -0.178 & -0.337 & -0.421 & 0.095 \\
\hline & \begin{tabular}{|l|} 
significance \\
(2-tailed)
\end{tabular} & - & 0.000 & 0.001 & 0.027 & 0.008 & 0.053 & 0.001 & 0.000 & 0.005 & 0.000 & 0.441 \\
\hline & sample size & 335 & 335 & 335 & 335 & 335 & 335 & 335 & 335 & 335 & 335 & 335 \\
\hline \multirow{3}{*}{ Level D } & $\begin{array}{l}\text { correlation } \\
\text { coefficient }\end{array}$ & 1.000 & 0.318 & -0.289 & 0.221 & 0.417 & 0.178 & -0.196 & -0.156 & -0.224 & -0.119 & 0.101 \\
\hline & \begin{tabular}{|l|} 
significance \\
(2-tailed)
\end{tabular} & - & 0.024 & 0.042 & 0.123 & 0.003 & 0.217 & 0.172 & 0.000 & 0.117 & 0.410 & 0.484 \\
\hline & sample size & 300 & 300 & 300 & 300 & 300 & 300 & 300 & 300 & 300 & 300 & 300 \\
\hline \multirow{3}{*}{ Level E } & $\begin{array}{l}\text { correlation } \\
\text { coefficient }\end{array}$ & 1.000 & 0.415 & -0.013 & 0.059 & 0.479 & 0.013 & -0.013 & -0.205 & -0.364 & -0.220 & 0.103 \\
\hline & \begin{tabular}{|l|} 
significance \\
(2-tailed)
\end{tabular} & - & 0.055 & 0.956 & 0.794 & 0.024 & 0.956 & 0.956 & 0.306 & 0.095 & 0.326 & 0.647 \\
\hline & sample size & 45 & 45 & 45 & 45 & 45 & 45 & 45 & 45 & 45 & 45 & 45 \\
\hline
\end{tabular}

PLOS was iteratively affected by multi-factors, so that the evaluation model should be a fuzzy rule with Multi Inputs and Single Output (MISO). The fuzzy neural network system based on Takagi-Sugeno (T-S) model was used to establish the evaluation model. It consisted of the premise network and the latter network, which were used to match the fuzzy rules (Cheng 2012). The system structure was illustrated in Figure 4.

The 1st floor of the premise network was the input floor. The connections were linked with each significant influencing factor of PLOS and passing the values of influencing factors $x=\left[x_{1}, x_{2}, \ldots, x_{n}\right]^{T}$ to the next floor. The total node of this floor $\mathrm{n}$ was the number of the influencing factors.

The 2nd floor of the premise network was the fuzzification floor. This floor was used to calculate the membership function $u_{i}^{j}$, which influenced PLOS. It could be expressed with Gaussian membership function as follows:

$$
u_{i}^{j}=u_{A_{i}^{j}}\left(x_{i}\right)=\exp \left(-\frac{\left(x_{i}-c_{i}^{j}\right)^{2}}{b_{i}^{j}}\right),
$$

where: $i=1,2, \ldots, n ; j=1,2, \ldots, m_{i} ; m_{i}$ was the number of fuzzy separation of $x_{i} ; c_{i}^{j}, b_{i}^{j}$ were the center and width of the membership function respectively; the total node of this floor was $\sum_{i=1}^{n} m_{i}$.

The 3rd floor of the premise network was the fuzzy rules floor. It was used to calculate the fitness value of each premise rule. The calculation was:

$$
\alpha_{j}=\min \left(u_{1}^{i_{1}}, u_{2}^{i_{2}}, \ldots, u_{n}^{i_{n}}\right) .
$$

On the other hand, calculate with the multiple multiplication operators:

$$
\alpha_{j}=u_{1}^{i_{1}} \cdot u_{2}^{i_{2}} \cdot \ldots \cdot u_{n}^{i_{n}},
$$

where: $i_{1} \in\left(1,2, \ldots, m_{1}\right) ; i_{2} \in\left(1,2, \ldots, m_{2}\right) ; \ldots ; i_{n} \in(1,2, \ldots$, $\left.m_{n}\right) ; j=1,2, \ldots, m ; m \in \sum_{i=1}^{n} m_{i}$; the total node of this floor
was $m$.

The 4th floor of the premise network was the normalization calculation. It was used to avoid the oscillating of the model caused by the difference of the magnitudes among each influencing factor. The total node of this floor 


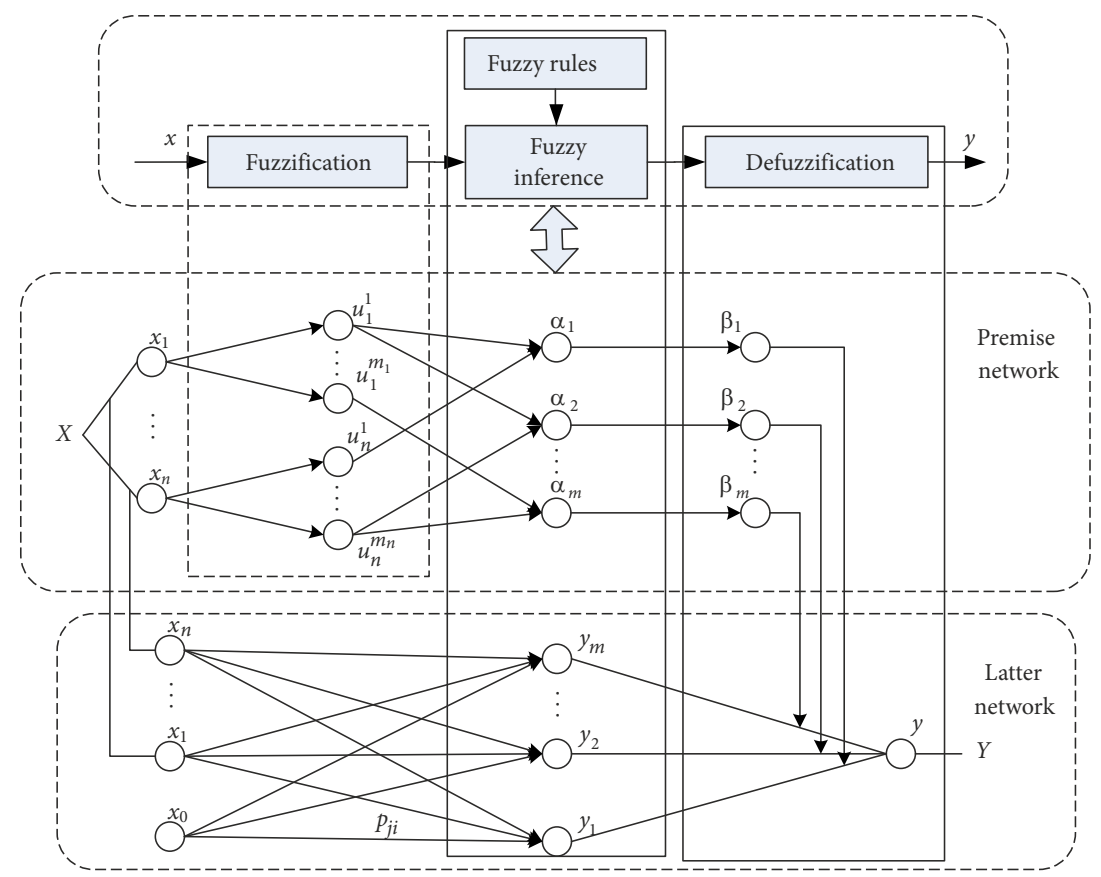

Figure 4 . The system structure of a fuzzy neural network based on T-S model

was the same as the 3 rd floor. The calculation was:

$$
\beta_{j}=\frac{\alpha_{j}}{\sum_{i=1}^{m} \alpha_{i}} .
$$

The 1st floor of the latter network was the input floor. The input value of node 0 was $x_{0}=1$. It provided the constant of the PLOS result.

The 2nd floor of the latter network was the fuzzy rules floor. It was used to calculate the fitness value of each latter rule. The calculation was:

$$
y_{j}=p_{j_{0}}+p_{j_{1}} \cdot x_{1}+\ldots+p_{j_{n}} \cdot x_{n}=\sum_{k=0}^{n} p_{j_{k}} \cdot x_{k}
$$

where: $k=1,2, \ldots, n ; j=1,2, \ldots, m$; the total node of this floor was $m$.

The 3rd floor of the latter network was the output floor. It was used to output the defuzzification result of PLOS. The calculation was:

$$
y=\sum_{j=1}^{m} \beta_{j} \cdot y_{j} .
$$

The structure of fuzzy neural network established here was a multilayer forward feedback network of local approximation. The network learning process can be achieved with the error Back Propagation (BP) algorithm. The error can be calculated as follows:

$$
e=\frac{1}{2} \cdot\left(y_{d}-y_{c}\right),
$$

where: $y_{d}$ was the grade of the actual surveyed PLOS; $y_{c}$ was the grade of calculated PLOS from the model.

The connective weights of the latter network can be calculated as:

$$
\begin{aligned}
& p_{i}^{j}(k)=p_{i}^{j}(k-1)-a \cdot \frac{\partial e}{\partial p_{i}^{j}}= \\
& p_{i}^{j}(k-1)-a \cdot\left(y_{d}-y_{c}\right) \cdot \frac{a^{j}}{\sum_{j=1}^{n} a^{j} \cdot x_{i}},
\end{aligned}
$$

where: $a$ was the learning-ratio of the network; $x_{i}$ was the input influencing factor of PLOS; $a^{j}$ was the multiple multiplication operator of the membership of the influencing factors.

The center and the width of the membership function were as follows respectively:

$$
\begin{aligned}
& c_{i}^{j}(k)=c_{i}^{j}(k-1)-\gamma \cdot \frac{\partial e}{\partial c_{i}^{j}} ; \\
& b_{i}^{j}(k)=b_{i}^{j}(k-1)-\gamma \cdot \frac{\partial e}{\partial b_{i}^{j}} .
\end{aligned}
$$

\subsection{The modelling result and accuracy test}

The models of PLOS were respectively established under each level of the pedestrian flow rate, and fuzzy neural network was used to train them. The input of the model was determined by the results of Table 5, which were the significant influencing factors of pedestrians' satisfaction under each level of pedestrian flow rate. The output of the model was PLOS on sidewalks. Since the original data was pedestrians' satisfaction ratings of 10 grades, it would transform into the PLOS of 6 grades. There is no clear boundary of different scores of pedestrians' satisfaction, so we used fuzzy clustering method to divide the grade. The result of fuzzy clustering is shown in Table 6. The scores of pedestrians' satisfaction grouped into 6 grades according to clustering centers and score threshold. 
Table 6. Comparison of pedestrians' satisfaction and PLOS

\begin{tabular}{|l|c|c|c|c|c|c|}
\hline PLOS & level A & level B & level C & level D & level E & level F \\
\hline Numbers compared to PLOS & 6 & 5 & 4 & 3 & 2 & 1 \\
\hline Clustering center & 9.14 & 7.91 & 7.24 & 5.98 & 5.12 & 3.25 \\
\hline Score threshold & {$[10,8.42)$} & {$[8.42,7.72)$} & {$[7.72,6.39)$} & {$[6.39,5.78)$} & {$[5.78,4.41)$} & {$[4.41,0)$} \\
\hline Scores of pedestrians' satisfaction & $10 \ldots 9$ & 8 & 7 & 6 & 5 & $4 \ldots 1$ \\
\hline
\end{tabular}

Through the self-adapted training of the fuzzy neural network, each evaluation result of PLOS and the error curves under level A to level D of pedestrian flow rate was obtained. Figure 5 showed the consequences. The horizontal axis shows the number of sample. The vertical axis shows the PLOS and the error. The curve of actual output represents the PLOS, which is transformed from pedestrians' satisfaction. The curve of modelling output represents the PLOS, which is calculated by fuzzy neural network. The curve of error represents the difference between actual output and modelling output.

It could be seen in Figure 5a and 5b that, when pedestrian flow rate was at level A or level B, the PLOS of the surveyed sidewalks with different road facility conditions and environmental conditions contained level A to level F. It meant that road facility conditions and environmental conditions significantly affected pedestrians' satisfaction when pedestrians walked under low density conditions. The PLOS could be raised by improving the road facility and environmental conditions of the sidewalks. Although the degree of freedom in walking was high under the conditions with poor road facility conditions and environmental conditions, the total level of service might still reduce to a very low level. In these situations, road facility conditions and environmental factors were the leading factors affecting PLOS on sidewalks.

As shown in Figure 5c and 5d, when pedestrian flow rate was at level C or level D, the PLOS of the surveyed sidewalks with different road facility conditions and environmental conditions just contained the levels below the ones of pedestrian flow rate. It meant that the influence of road facility conditions and environmental conditions impacted on pedestrians' satisfaction weakened when pedestrians walked under high density conditions. The pedestrian flow rate became the leading influencing factors. PLOS on sidewalks almost could not be raised by improving the road facility conditions and environmental conditions. However, the worse road facility conditions and environmental conditions could still bring down the total PLOS. In these situations, PLOS on sidewalks was affected by both pedestrian flow rate and other factors and the impact of pedestrian flow rate was more significant.

When pedestrian flow rate came down to level $\mathrm{E}$, the significant influencing factor was only the segregated facilities between pedestrians and vehicles. In this sense, after pedestrian volume increased to the crowded condition, pedestrians almost didn't concern about road facility conditions and environmental conditions any more. The pedestrian flow rate affected PLOS as the leading factor.
If there was no segregation between pedestrians and vehicles, the level of service would drop until the lowest level. Thus, PLOS E and F on sidewalks could be classified directly based on the level of pedestrian flow rate and the segregation between pedestrians and vehicles. It was unnecessary to establish the fuzzy neural network models for these 2 levels.

The accuracy of the fuzzy neural network could be seen from the error curves in Figure 5. The expected output results were the integer values from 1 to 6 , which were transformed from pedestrians' satisfaction. However, the actual output results were calculated based on the selfadapted relationship between pedestrians' satisfaction and the influencing factors, which might not be integer. Thus, if the error was in the interval of $[-0.5,0.5]$, the result of the model was completely the same with the actual level. Furthermore, PLOS was obtained based on pedestrians' satisfaction. Due to the influence of the difference among pedestrians' feelings, there existed the overlap parts to the scores of adjacent levels. The degree of pedestrians' satisfaction scores belonging to each PLOS was similar to the membership function. Therefore, if the error was in the interval of $[-1,1]$, the result of the model could be regarded to be consistent with the actual level. In Figure 5a, some of the errors reach to 2 , it is because different pedestrians perceive the same sidewalk differently and different pedestrians have different evaluating criterion. However, only $3.2 \%$ of samples' errors reach to $2,96.8 \%$ of samples' errors are in the interval of $[-1,1]$, so the effect on the accuracy of the evaluation models seems limited.

Based on the analysis above, the accuracy of the evaluation models under the pedestrian flow rate from level A to level D was $80 \%, 81 \%, 93 \%$ and $100 \%$ respectively. The average accuracy of the 6 levels was $92 \%$. With the increase of the pedestrian flow rate, the influencing factors of the road facility conditions and environmental conditions of PLOS decreased. The accuracy of the model was improved along with it. When the PLOS went down to below level $\mathrm{D}$, the error of the model almost disappeared.

The PLOS on the sidewalks surveyed in this research was evaluated with the models in the existing research. Bian et al. (2007) gathered all the influencing factors on pedestrians' satisfaction to establish an integrated model with stepwise linear regression method. Petritsch et al. (2008) established the models based on pedestrian density and other factors respectively. The overall PLOS was the worse of the 2 levels. With calculation, the accuracy of the evaluation model in Petritsch's research was 0.79 and the accuracy of the evaluation model in Bian's research was 0.76. 
a)

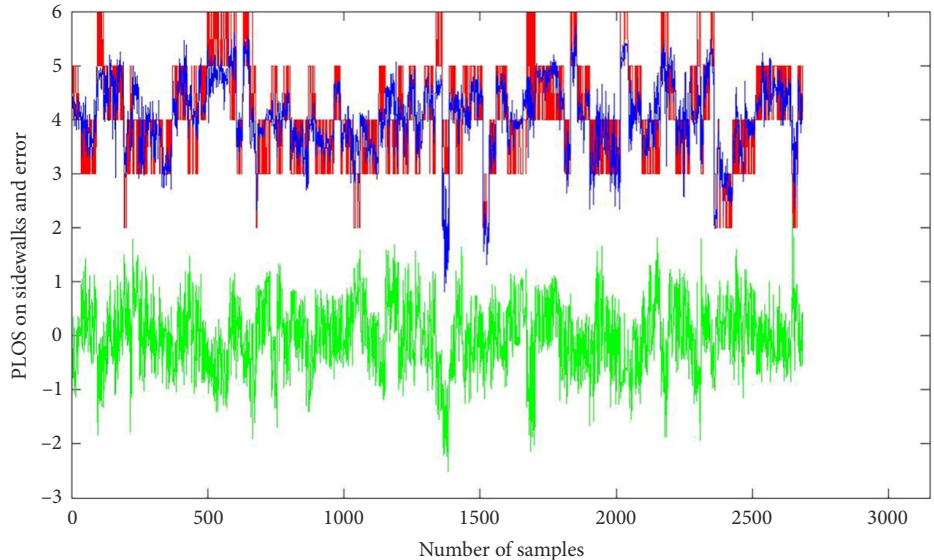

b)

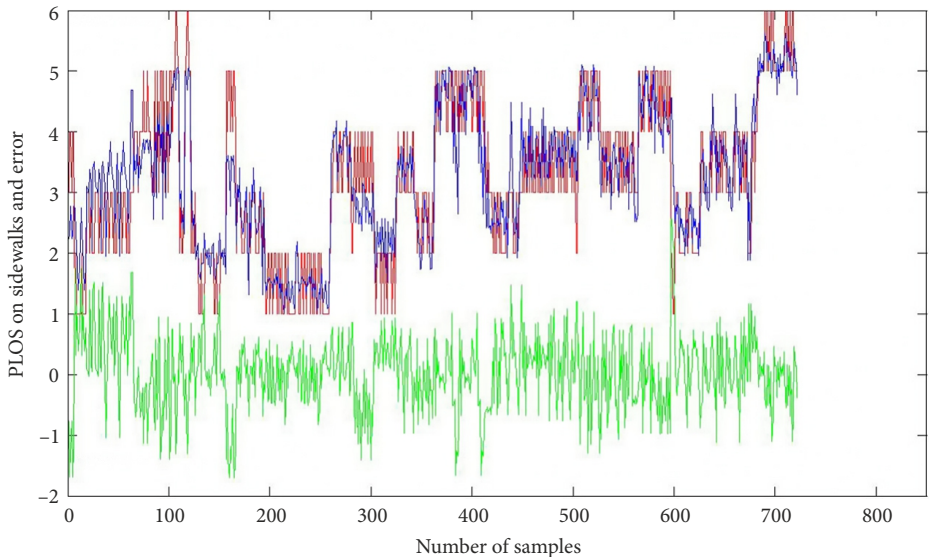

c)

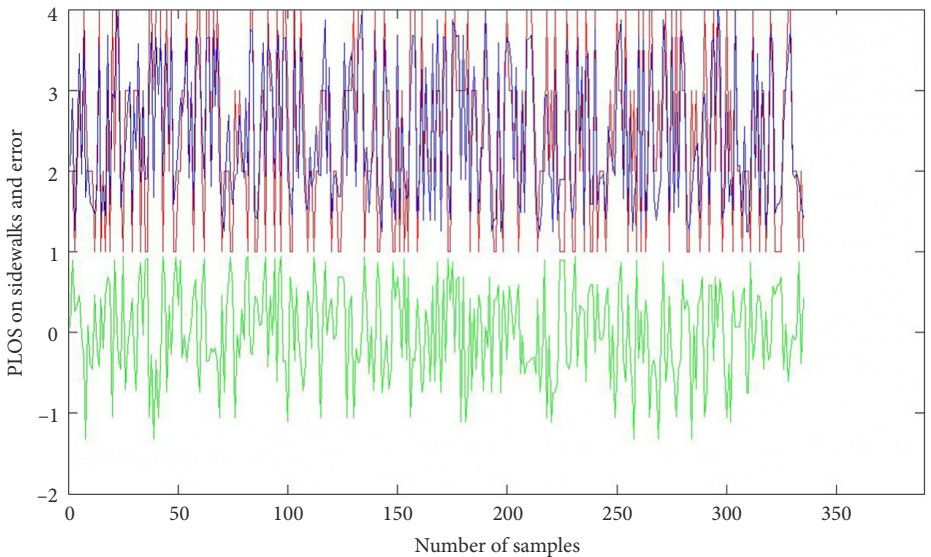

d)

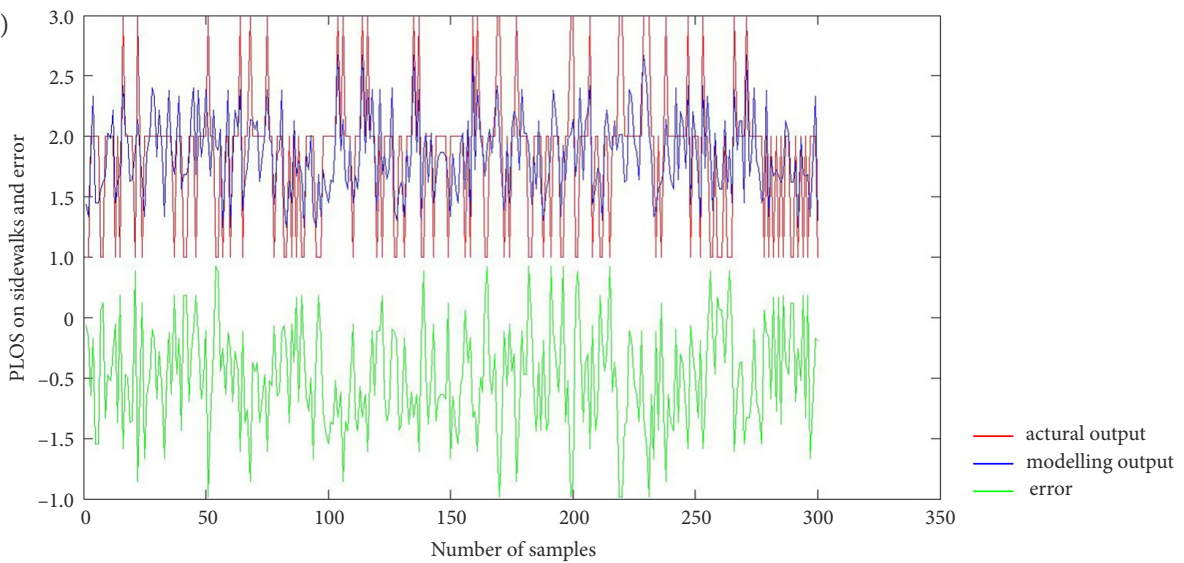

Figure 5. The evaluation (modelling) results of multi-factors affected PLOS and the error curves: a - at level A of pedestrian flow rate; $\mathrm{b}$ - at level $\mathrm{B}$ of pedestrian flow rate; $\mathrm{c}$ - at level $\mathrm{C}$ of pedestrian flow rate; $\mathrm{d}$ - at level $\mathrm{D}$ of pedestrian flow rate 
It could be seen that the accuracy of the PLOS model established with the fuzzy neural network method on the basis of classifying the levels of pedestrian flow rate (0.92) had been greatly improved comparing with the existing models. This showed the method in this research could evaluate PLOS on sidewalks in a more factual and accurate way.

\section{Conclusions}

This paper improved the shortcoming in the existing evaluation methods for PLOS, such as the strong subjectivity and inability to present pedestrians' psychological demand. Pedestrians' walking behaviours were caught with video recording and image tracking technology. Pedestrians' psychological demand was presented with the index of pedestrians' relative lateral displacement in case of bypassing and avoiding behaviours. The pedestrian flow rate was classified objectively according to different pedestrians' traffic states

Pedestrians' satisfaction questionnaires were conducted with intercept survey method from the perspective of pedestrians' sense of safety and comfort. Significant influencing factors of pedestrians' satisfaction under different levels of pedestrian flow rate were extracted through Spearman rank correlation method. The evaluation index system was also built under different levels of pedestrian flow rate. The influencing degree of different kinds of factors was shown more scientifically and visually.

Based on the threshold classification of pedestrian flow rate, the evaluation models for PLOS on sidewalks with multi-factors under different pedestrian flow rates were established respectively. Fuzzy neural network method was used to train the models and the average accuracy of the models was 0.92 , which was higher than the accuracy of the existing models. This evaluation method could evaluate PLOS on sidewalks more accurately. The model had strong adaptability because of the learning capability and data driven features of the neural network itself. With the samples increase, the scope of its application can be further expanded by using the measure of regularly relearning.

\section{Funding}

This work was supported by National Natural Science Foundation of China (Grant No 51108012).

\section{Author contributions}

Shinan Shu conducted the study and wrote the paper.

Yang Bian and Lin Zhao jointly performed statistical analysis.

Jian Rong and Xiaoming Liu established the model.

All authors contributed to the survey project and revision of the paper.

All authors read and approved the final paper.

\section{Disclosure statement}

The authors declare that they have no competing interests.

\section{References}

Bian, Y.; Wang, W.; Lu, J.; Ma, J.; Tan, D. 2007. Pedestrian level of service for sidewalks in China, in TRB 86th Annual Meeting Compendium of Papers CD-ROM, DC, 21-25 January 2007, Washington, DC, US, 1-24.

Chen, S.-H.; Wu, C.-C.; Li, P.-Y.; Paramitha, P. A. 2017. Evaluation of pedestrian transportation facilities in Taiwan using linear regression and support vector regression, Road Materials and Pavement Design 18(Sup 3): 170-179.

https://doi.org/10.1080/14680629.2017.1329872

Cheng, F. H. 2012. A Study on Air Quality Assessment Applied BP Neural Network and Fuzzy Neural Networks. MSc Thesis. Guangdong University of Technology, Guangzhou, China. 97 p. (in Chinese).

CHTS. 1998. Traffic Engineering Manual. China Highway and Transportation Society (CHTS). China Communications Press, Beijing, China. (in Chinese).

Dixon, L. B. 1996. Bicycle and pedestrian level-of-service performance measures and standards for congestion management systems, Transportation Research Record: Journal of the Transportation Research Board 1538: 1-9.

https://doi.org/10.1177/0361198196153800101

Dowling, R.; Flannery, A.; Landis, B.; Petritsch, T.; Rouphail, N.; Ryus, P. 2008. Multimodal level of service for urban streets, Transportation Research Record: Journal of the Transportation Research Board 2071: 1-7. https://doi.org/10.3141/2071-01

Fruin, J. J. 1971. Pedestrian Planning and Design. Metropolitan Association of Urban Designers and Environmental Planners. $206 \mathrm{p}$.

Fu, D.; Feng, W.-D.; Yu, Q.-F.; Xu, Y.-D. 2008. Linear self-calibration method for camera, Opto-Electronic Engineering 35(1): 71-75. (in Chinese).

Guo, H.; Zhao, F.; Wang, W.; Zhou, Y.; Zhang, Y.; Wets, G. 2014. Modeling the perceptions and preferences of pedestrians on crossing facilities, Discrete Dynamics in Nature and Society 2014: 949475. https://doi.org/10.1155/2014/949475

Kadali, B. R.; Vedagiri, P. 2015. Evaluation of pedestrian crosswalk level of service (LOS) in perspective of type of landuse, Transportation Research Part A: Policy and Practice 73: 113-124. https://doi.org/10.1016/j.tra.2015.01.009

Kang, L.; Xiong, Y.; Mannering, F. L. 2013. Statistical analysis of pedestrian perceptions of sidewalk level of service in the presence of bicycles, Transportation Research Part A: Policy and Practice 53: 10-21. https://doi.org/10.1016/j.tra.2013.05.002

Khisty, C. J. 1994. Evaluation of pedestrian facilities: beyond the level-of-service concept, Transportation Research Record: Journal of the Transportation Research Board 1438: 45-50.

Kim, S.; Choi, J.; Kim, S.; Tay, R. 2014. Personal space, evasive movement and pedestrian level of service, Journal of Advanced Transportation 48(6): 673-684. https://doi.org/10.1002/atr.1223

Kim, S.; Choi, J.; Kim, Y. 2011. Determining the sidewalk pavement width by using pedestrian discomfort levels and movement characteristics, KSCE Journal of Civil Engineering 15(5): 883-889. https://doi.org/10.1007/s12205-011-1173-1

Landis, B. W.; Petritsch, T. A.; McLeod, P. S.; Huang, H. F.; Guttenplan, M. 2005. Video simulation of pedestrian crossings at signalized intersections, Transportation Research Record: Journal of the Transportation Research Board 1920: 49-55. https://doi.org/10.1177/0361198105192000106 
Landis, B. W.; Vattikuti, V. R.; Ottenberg, R. M.; McLeod, D. S.; Guttenplan, M. 2001. Modeling the roadside walking environment: pedestrian level of service, Transportation Research Record: Journal of the Transportation Research Board 1773: 82-88. https://doi.org/10.3141/1773-10

Li, X.; Guo, F.; Kuang, H.; Zhou, H. 2017. Effect of psychological tension on pedestrian counter flow via an extended cost potential field cellular automaton model, Physica A: Statistical Mechanics and its Applications 487: 47-57. https://doi.org/10.1016/j.physa.2017.05.070

Liang, K. 2006. Study on Fuzzy Evaluation Mode of Levels of Service for Sidewalks. MSc Thesis. Huazhong University of Science and Technology, Wuhan, Hubei, China. (in Chinese).

Muraleetharan, T.; Hagiwara, T. 2007. Overall level of service of urban walking environment and its influence on pedestrian route choice behavior: analysis of pedestrian travel in Sapporo, Japan, Transportation Research Record: Journal of the Transportation Research Board 2002: 7-17. https://doi.org/10.3141/2002-02

Myers, J. L.; Well, A. D. 2003. Research Design and Statistical Analysis. Psychology Press. 736 p. https://doi.org/10.4324/9781410607034

Petritsch, T. A.; Landis, B. W.; Huang, H. F.; Dowling, R. 2008. Pedestrian level-of-service model for arterials, Transportation Research Record: Journal of the Transportation Research Board 2073: 58-68. https://doi.org/10.3141/2073-07

Petritsch, T, A; Landis, B. W.; McLeod, P. S.; Huang, H. F.; Challa, S.; Skaggs, C. L.; Guttenplan, M.; Vattikuti, V. 2006. Pedestrian level-of-service model for urban arterial facilities with sidewalks, Transportation Research Record: Journal of the Transportation Research Board 1982: 84-89. https://doi.org/10.1177/0361198106198200111

Roper, J. A. M.; Hassan, S. E. 2014. How do vision and hearing impact pedestrian time-to-arrival judgments?, Optometry and Vision Science 91(3): 303-311. https://doi.org/10.1097/OPX.0000000000000161

Sahani, R.; Bhuyan, P. K. 2017. Pedestrian level of service criteria for urban off-street facilities in mid-sized cities, Transport 32(2): 221-232. https://doi.org/10.3846/16484142.2014.944210

Shan, X.; Ye, J.; Chen, X. 2016. Proposing a revised pedestrian walkway level of service based on characteristics of pedestrian interactive behaviours in China, Promet - Traffic \& Transportation 28(6): 583-591. https://doi.org/10.7307/ptt.v28i6.1947

Sieben, A.; Schumann, J.; Seyfried, A. 2017. Collective phenomena in crowds - where pedestrian dynamics need social psychology, Plos One 12(6): e0177328.

https://doi.org/10.1371/journal.pone.0177328

Steiner, R. L.; Landis, B. W.; Miller, D. W. 2002. Refinement of the Roadway Segment Level of Service. Florida Department of Transportation (FDOT), Tallahassee, FL, US. 15 p. Available from Internet: https://www.fsutmsonline.net/images/uploads/ reports/fdot_bc354_45_rpt.pdf

Tang, M. 2010. Research on Pedestrian Traffic Behavior Model and Simulation Algorithm in Passenger Transfer Hub. $\mathrm{PhD}$ Thesis. Jilin University, Jilin, China. (in Chinese).

TRB. 2010. Highway Capacity Manual. Transportation Research Board (TRB), Washington, DC, US. 1650 p.

Wahba, M. A.; Bridwell, L. G. 1976. Maslow reconsidered: a review of research on the need hierarchy theory, Organizational Behavior and Human Performance 15(2): 212-240. https://doi.org/10.1016/0030-5073(76)90038-6

Zhang, Z.; Jia, L.; Qin, Y. 2016. Level-of-service based hierarchical feedback control method of network-wide pedestrian flow, Mathematical Problems in Engineering 2016: 9617890.

https://doi.org/10.1155/2016/9617890 